\title{
A NEW METHOD FOR CALCULATING WAVE GENERATION AND PROPAGATION IN A CURVED SPACE-TIME
}

By modifying the Sobolev method of solving the Cauchy problem, a new formalism for calculating wave generation in a curved space-time has been developed. An integral equation equivalent to a wave equation with variable coefficients is given and its physical content is discussed. The new formalism is valid in any admissible coordinate system.

\section{Introduction}

In the present paper we are going to propose a new method for calculating electromagnetic and gravitational wave generation through sources influenced by background gravitational fields and to tackle the problem of wave propagation. Electromagnetic and gravitational radiations are considered by us as small perturbations, and their self-action on metric is neglected. We also assume that the problem is separable, i. e., we have equations, each of which contains only one unknown function. Thus we are going to discuss a problem that can be formulated mathematically as integration of a differential equation of a normal hyperbolic type *

$$
\begin{aligned}
& \hat{L}_{\varphi}=4 \pi \varrho(x), \\
& \hat{L}_{\varphi} \equiv g^{\alpha \beta} \varphi_{, \alpha \beta}+b^{\alpha} \varphi_{, \alpha}+C \varphi,
\end{aligned}
$$

where $g^{\alpha \beta}, b^{\alpha}$ and $C$ are the given functions of the coordinates $x \equiv$ $\equiv\left(x^{0}, x^{1}, x^{2}, x^{3}\right)$ and $\varrho(x)$ is source density. J. Hadamard [ [ $\left.{ }^{1}\right]$ has proved that the Cauchy data on an open space-like hypersurface uniquely determine the solution of equation (1). By making use of his ideas, B. DeWitt and R. Brehme $\left[{ }^{2}\right]$ have elaborated the Green function approach to electromagnetic radiation in a curved space-time. Another solution of the Cauchy problem has been proposed by S. Sobolev $\left[{ }^{3}\right]$. In the present paper we shall make use of the ideas of the last-mentioned author for deriving new wave-generation formulae. We shall demonstrate that if the coefficients in equation (1) depend on coordinates, the well-known retarded solution of the flat space-time wave equation

$$
\varphi_{\text {flat }}\left(x_{0}\right)=\int \frac{[\varrho]}{R} d V+\text { a surface integral }
$$

* Greek indices take values $0,1,2,3$, Latin indices $-1,2,3$, summation over repeated indices is assumed; $f_{, \alpha} \equiv \frac{\partial f}{\partial x \alpha}$. 
takes the form of the integral equation

$$
\varphi\left(x_{0}\right)=\int\left[S_{0}\right] d V-\frac{1}{4 \pi} \int\left[\varphi(x) \hat{M}^{*} S\right] d V+\text { a surface integral. }
$$

The quantities in square brackets are to be evaluated on a light cone that has a vertex at point $x_{0} ; S$ is a two-point function, which we propose to call the Sobolev function, $\hat{M}^{*}$ is a differential operator. In the case of the Cauchy problem one is interested in the dependence of the solution on the surface integral containing the Cauchy data. In the case of wave generation the volume integral with the source term 0 is of prime interest. The first and the third term on the right-hand side of integral equation (3) give us direct radiation, the second term yields scattered (reflected) radiation. The solution of equation (3) can be evaluated by successive steps, the zeroth-approximation solution being direct radiation.

Our formalism is valid in any admissible coordinate system, while that of S. Sobolev has been elaborated in a semi-geodesic coordinate system. A generalization of the Sobolev method to a system of equations has been given by $M$. Chevalier $\left[{ }^{4}\right]$, who also applies semi-geodesic coordinates.

The paper is arranged as follows. The second section will review wellknown formulae that will be used later. The main ideas related to the Green function approach will be discussed to elucidate differences between the Green function and the Sobolev one and differences between the corresponding methods. Fundamental formulae of the new formalism will be derived in the third section, and their physical interpretation will be given in the fourth section. The fifth section will deal with the Maxwell equations in the Schwarzschild background.

\section{Adjoint Differential Operators, the Green Formula and the Green Function}

Let us begin with definitions that can be found in monographs by J. Hadamard $\left[{ }^{1}\right]$ and by S. Sobolev $\left[{ }^{5}\right]$. The adjoint operator $\hat{L}^{*}$ of the differential operator (2) is defined as follows:

We have

$$
\hat{L}^{*} \psi \equiv\left(g^{\alpha \beta} \psi\right)_{, \alpha \beta}-\left(b^{\alpha} \psi\right)_{, \alpha}+C \psi .
$$

$$
\begin{gathered}
\psi \hat{L} \varphi-\varphi \hat{L}^{*} \psi=P_{, \alpha}^{\alpha}, \\
P^{\alpha} \equiv \psi g^{\alpha \beta} \varphi, \beta-\varphi\left(g^{\alpha \beta} \psi\right)_{, \beta}+b^{\alpha} \varphi \psi .
\end{gathered}
$$

The same formulae are valid in $\mathrm{n}$-dimensional space. By putting $g^{\alpha \beta}=0$ in (2) and (4), one obtains the definition of the first-order adjoint differential operator that will be used in the third section.

By making use of the Gauss-Ostrogradsky formula, one obtains from (5) the following relation:

$$
\int_{\Omega^{*}}\left(\psi \hat{L} \varphi-\varphi \hat{L}^{*} \psi\right) d^{4} x=-\int_{\Sigma^{3}} P^{\alpha} d f_{\alpha},
$$

where $d f_{\alpha}$ is an element of the three-dimensional boundary $\Sigma^{3}$ of the fourdimensional region $\Omega^{4}$.

The Green function $G\left(x, x_{0}\right)$ is usually defined as the solution of the differential equation

$$
\hat{L}^{*} G=4 \pi \delta\left(x-x_{0}\right)
$$


which satisfies certain boundary conditions and vanishes outside the past light cone drawn from point $x_{0}$. By replacing $\psi$ with $G$ in identity (7) and by making use of equation (1), we obtain the Green function representation of the solution

$$
\varphi\left(x_{0}\right)=\int_{\Omega^{4}} G\left(x, x_{0}\right) \varrho(x) d^{4} x+\frac{1}{4 \pi} \int_{\Sigma^{3}} P^{\alpha} d f_{\alpha} .
$$

Here the first integral on the right-hand side is taken over the whole past history of the source. We note that the Green function for electromagnetic radiation in the weak Schwarzschild field has been evaluated and used by C. Morette DeWitt and B. DeWitt $\left[{ }^{6}\right]$ and by P. C. Peters $\left[{ }^{7}\right]$.

One of the difficulties arising in the case of the Green function approach is the determination of the Green function itself, the integration of the adjoint equation (8). As a rule, this can be performed by a method of successive approximations, e. g., the scheme used by J. Hadamard to determine the fundamental solution consists in the integration of a set of recurrent equations. We insist on solving the problem by iterating an integral equation instead.

\section{Fundamental Formulae and the Sobolev Function}

Preserving the terminology proposed by S. Sobolev $\left[{ }^{3}\right]$, we shall call integral equation (3) the first fundamental formula, and its solution in the form of the recurrent system given at the end of this section will be called the second fundamental formula. Before their derivation we shall give transformation laws for the coefficients in equation (1) and shall discuss the problem of determining the light cone.

3.1. Suppose that $g^{\alpha \beta}$ and $b^{\alpha}$ are regular functions and have derivatives up to a certain order. In general relativity we have equations of type (1), where $g^{\alpha \beta}$ is a component of a metric tensor, a function of class $C^{1}$ and piecewise of class $C^{3}$ (A. Lichnerowicz $\left.\left[{ }^{8}\right]\right)$. After having introduced new independent variables

$$
\begin{aligned}
& \tilde{x}^{\alpha}=\tilde{x}^{\alpha}\left(x^{\beta}\right), \\
& x^{\beta}=x^{\beta}\left(\tilde{x}^{\alpha}\right),
\end{aligned}
$$

equation (1) assumes the following form:

$$
\begin{aligned}
& \hat{L}_{\varphi}\left(\tilde{x}^{\alpha}\right)=4 \pi \varrho\left(\tilde{x}^{\alpha}\right), \\
& \hat{L} \varphi \equiv \widetilde{g}^{\alpha \beta} \frac{\partial^{2} \varphi}{\partial \widetilde{x}^{\alpha} \partial \tilde{x}^{\beta}}+\widetilde{b}^{\alpha} \frac{\partial \varphi}{\partial \widetilde{x}^{\alpha}}+C \varphi, \\
& \tilde{g}^{\alpha \beta}=\frac{\partial \tilde{x}^{\alpha}}{\partial x^{\sigma}} \frac{\partial \widetilde{x}^{\beta}}{\partial x^{\rho}} g^{\sigma \rho}, \\
& \widetilde{b}^{\alpha}=\frac{\partial^{2} \widetilde{x}^{\alpha}}{\partial x^{\sigma} \partial x^{\rho}} g^{\sigma \rho}+\frac{\partial \widetilde{x}^{\alpha}}{\partial \mathrm{x}^{\sigma}} b^{\sigma}
\end{aligned}
$$

Let $\widetilde{x}^{0} \equiv u$ be a characteristic coordinate. Then by definition $\widetilde{g}^{00}=0$, and in old coordinates

$$
g^{\alpha \beta} u_{, \alpha} u_{, \beta}=0
$$

Introducing the notation $\dot{\varphi} \equiv \frac{\partial \varphi}{\partial u}$ and putting $\tilde{x}^{i}=x^{i}$, we have

$$
\hat{L} \varphi=M \varphi+\hat{N} \dot{\varphi}
$$




$$
\begin{aligned}
& \hat{M} \varphi \equiv g^{i k} \varphi_{\mid i k}+b^{i} \varphi_{\mid i}+C \varphi, \\
& \hat{N} \dot{\varphi} \equiv 2 \widetilde{g}^{0 i} \dot{\varphi}_{\mid i}+\widetilde{b}^{0} \dot{\varphi} .
\end{aligned}
$$

Here $\varphi_{\mid i}$ is an inner derivative on the light cone, i. e., $\varphi_{\mid i} \equiv \varphi_{, i \mid u=\text { const }}$. In the case of the arbitrary time coordinate $t u=u\left(t, x^{i}\right)$ and

$$
\varphi_{, i}=\varphi_{\mid i}+\dot{\varphi} u_{, i} .
$$

Next we shall make a few notes on the determination of a characteristic conoid or light cone, as it is usually called in physics.

3.2. Let us find an integral of equation (14)

$$
u\left(x, x_{0}\right)=0 .
$$

Speaking geometrically, we shall construct a light cone $I^{-}\left(x_{0}\right)$, with the vertex at point $x_{0}$ (see Figure). Let

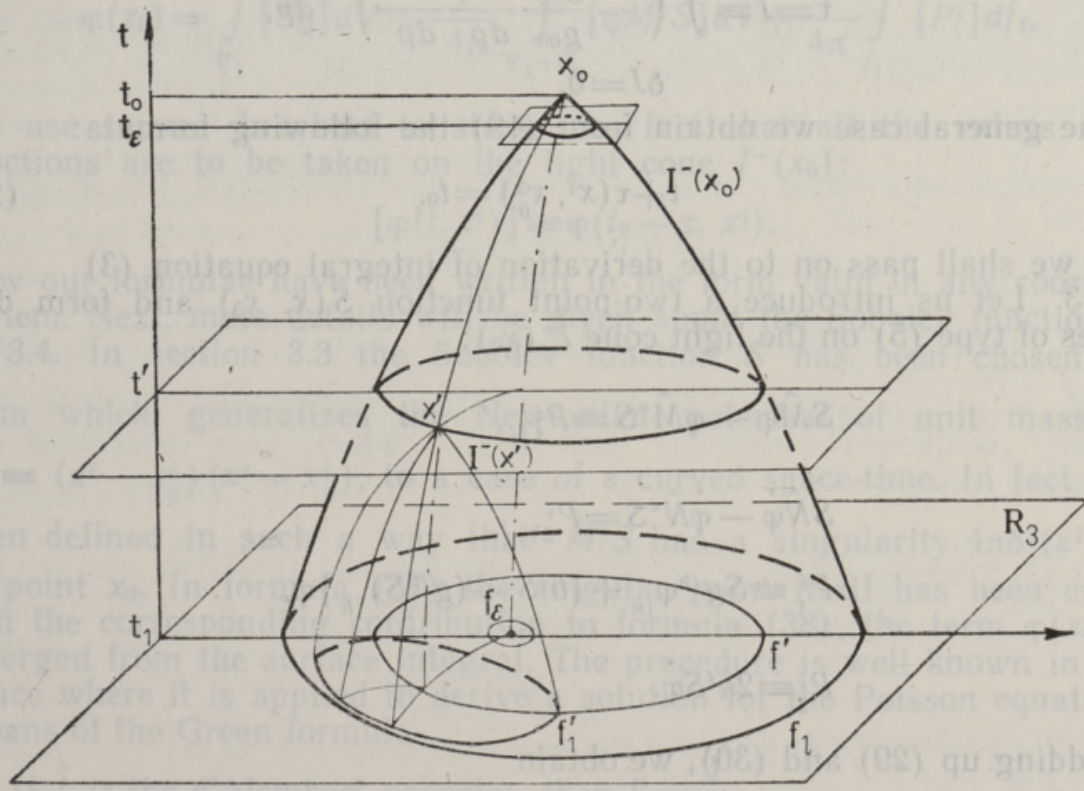

The map of past light cones $I^{-}\left(x_{0}\right)$ and $I^{-}\left(x^{\prime}\right)$ with one space dimension suppressed. Projections of intersections of $I^{-}\left(x_{0}\right)$ and coordinate surfaces $t=t_{\varepsilon}, t=t^{\prime}$ onto $R_{3}$, i. e., onto the coordinate surface $t=t_{1}$.

$$
\begin{aligned}
& k_{\alpha} \equiv u_{, \alpha}, \\
& k^{\alpha} \equiv g^{\alpha \sigma} k_{\sigma} .
\end{aligned}
$$

The characteristic system of equation $(14)\left(\left[{ }^{9}\right], \S 38\right)$ can be written as

$$
\begin{gathered}
\frac{d x^{0}}{k^{0}}=\frac{d x^{1}}{k^{1}}=\frac{d x^{2}}{k^{2}}=\frac{d x^{3}}{k^{3}}=d p, \\
\frac{d k_{\alpha}}{-\frac{1}{2} g_{, \alpha}^{\mu v} k_{\mu} k_{v}}=d p .
\end{gathered}
$$

In the latter expression there is no summation over $\alpha$. A great deal of 
work has been done on the determination of light cones. Only some results will be reproduced here. Suppose that a solution of equations (22) and (23) has been found:

$$
\begin{aligned}
& x^{\alpha}=x^{\alpha}\left(x_{0}^{\alpha}, k_{\alpha}^{0}, p\right), \\
& k_{\alpha}=k_{\alpha}\left(x_{0}^{\alpha}, k_{\alpha}^{0}, p\right),
\end{aligned}
$$

where $p$ is a parameter along a bicharacteristic and $k_{\alpha}^{0}$ is the value of $k_{\alpha}$ at point $x_{0}$, i. e., at the vertex of the light cone $I^{-}\left(x_{0}\right)$. By eliminating $k_{\alpha}^{0}$ and $p$ from equations (24) and (25), we obtain formula (19). In a static field the equation of the light cone can be presented in the following form:

$$
t+\tau\left(x^{i}, x_{0}^{i}\right)=t_{0}
$$

and $\tau$ can easily be found by making use of the variational principle (see $\left.\left[{ }^{10}\right]\right)$

$$
\begin{gathered}
\tau=J \equiv \int\left(-\frac{g_{i k}}{g_{00}} \frac{d x^{i}}{d p} \frac{d x^{k}}{d p}\right)^{1 / 2} d p, \\
\delta J=0 .
\end{gathered}
$$

In the general case we obtain from (19) the following formula:

$$
t+\tau\left(x^{i}, x_{0}^{\alpha}\right)=t_{0} .
$$

Now we shall pass on to the derivation of integral equation (3).

3.3. Let us introduce a two-point function $S\left(x, x_{0}\right)$ and form differences of type (5) on the light cone $I^{-}\left(x_{0}\right)$ :

$$
\begin{aligned}
& S \hat{M} \varphi-\varphi \hat{M}^{*} S=P_{1 \mid i}^{i}, \\
& S \hat{N} \dot{\varphi}-\dot{\varphi} \hat{N}^{*} S=P_{2 \mid i}^{i}, \\
& P_{1}^{i} \equiv S g^{i k} \varphi_{\mid k}+\left\{b^{i} S-\left(g^{i k} S\right)_{\mid k}\right\} \varphi, \\
& P_{2}^{i} \equiv 2 k^{i} \dot{S} \dot{.}
\end{aligned}
$$

By adding up (29) and (30), we obtain

$$
\begin{aligned}
& S \hat{L} \varphi-\varphi \hat{M}^{*} S-\dot{\varphi} \hat{N}^{*} S=P_{\mid i}^{i}, \\
& P^{i} \equiv P_{1}^{i}+P_{2}^{i} .
\end{aligned}
$$

Now we choose $S$ in the form of a solution of a differential equation and impose a boundary condition on it:

$$
\begin{aligned}
& \hat{N}^{*} S=0, \\
& \lim _{\varepsilon \rightarrow 0} \int_{\varepsilon} P^{i} d f_{i}=-4 \pi \varphi\left(x_{0}\right) .
\end{aligned}
$$

Here $f_{\varepsilon}$ denotes an infinitesimal surface around point $x_{0}$ in a space of parameters. Integrals of equation (35) and a more suitable form of boundary conditions to be satisfied by $S$ will be dealt with in section 3.4.

Let us parametrize points on the light cone $I^{-}\left(x_{0}\right)$ by ascribing to them the values of the coordinates of the points in three-space $x^{0} \equiv t=$ 
$=t_{1}=$ const via a one-to-one correspondence of the points on the same coordinate lines $x^{i}=$ const. $\left(x^{0} \neq u\right)$. By forming an integral on the light cone $I^{-}\left(x_{0}\right)$, by using expression (33) as an integrand, by taking into account condition (35) and by applying the Gauss-Ostrogradsky formula, we obtain

$$
\int_{V_{1}-V_{\varepsilon}}\left(S \hat{L} \varphi-\varphi \hat{M}^{*} S\right) d V=-\int_{f_{1}+f_{\varepsilon}} P^{i} d f_{i}
$$

Here $V_{1}-V_{\varepsilon}$ is the volume in the space of the parameters that enumerate the points on the light cone $I^{-}\left(x_{0}\right)$, between the coordinate surfaces $t=t_{1}$ and $t=t_{0}-\varepsilon \equiv t_{\varepsilon}$ (see Figure); $f_{1}$ is a two-dimensional surface formed by the intersection of the coordinate surface $t=t_{1}$ and the light cone $I^{-}\left(x_{0}\right), f_{\varepsilon}$ is the projection of the intersection of the coordinate surface $t=t_{\varepsilon}$ and $I^{-}\left(x_{0}\right)$ into the space of parameters. If $\varepsilon \rightarrow 0$, we obtain from (36) and (37) our first fundamental formula, an integral equation for $\varphi(x)$ :

$$
\varphi\left(x_{0}\right)=\int_{V_{1}}\left[S_{\varrho}\right] d V-\frac{1}{4 \pi} \int_{V_{1}-V_{\varepsilon}}\left[\varphi \hat{M}^{*} S\right] d V+\frac{1}{4 \pi} \int_{f_{1}}\left[P^{i}\right] d f_{i} .
$$

We use square brackets to stress the fact that all the values of the functions are to be taken on the light cone $I^{-}\left(x_{0}\right)$ :

$$
\left[\varphi\left(t, x^{i}\right)\right] \equiv \varphi\left(t_{0}-\tau, x^{i}\right) .
$$

Now our formulae have been written in the form valid in any coordinate system. Next, more details will be given about the Sobolev function.

3.4. In section 3.3 the Sobolev function $S$ has been chosen in a form which generalizes the Newtonian potential of unit mass $\frac{1}{R}$, $R^{2} \equiv\left(x^{i}-x_{0}^{i}\right)\left(x^{i}-x_{0}^{i}\right)$, to a case of a curved space-time. In fact it has been defined in such a way that $\hat{M}^{*} S$ has a singularity $4 \pi \delta^{3}\left(x^{i}-x_{0}^{i}\right)$ at point $x_{0}$. In formula (37) the singular point itself has been cut out and the corresponding contribution to formula (38), the term $\varphi\left(x_{0}\right)$ has emerged from the surface integral. The procedure is well known in a flat space where it is applied to derive a solution for the Poisson equation by means of the Green formula.

If $\hat{L}$ is the d'Alembert operator, then $S=\frac{1}{R}$,

and

$$
\hat{M}^{*} S=4 \pi \delta^{3}\left(x^{i}-x_{0}^{i}\right)
$$

$$
\hat{N}^{*} S=0
$$

In a curved space-time the conditions (39) and (35) may become contradictory, and we have kept the second equation and abandoned the first one.

Next we integrate equation (35) and then write the boundary conditions (36) at $f_{\varepsilon}$ in a form suitable for practical applications.

Equation $\hat{N}^{*} S=0$ can be written in the following form:

$$
\begin{aligned}
& k^{0} S_{, 0}+k^{i} S_{, i}+A S=0, \\
& A \equiv A_{0}+A_{1},
\end{aligned}
$$




$$
\begin{aligned}
& A_{0} \equiv k^{\alpha} ; \alpha-\frac{1}{2}\left(g^{\sigma \rho} k_{\sigma, \rho}+b^{\alpha} k_{\alpha}\right), \\
& A_{1} \equiv-\frac{\partial \ln \sqrt{-g}}{\partial x^{\alpha}} k^{\alpha}-k^{0}, 0-k^{i}{ }_{, 0} k_{i} \frac{1}{k_{0}} .
\end{aligned}
$$

Here the semicolon denotes covariant differentiation, $g \equiv \operatorname{det} g_{\mu v}$, $g_{\mu v}$ is the matrix reciprocal to $g^{\mu v}$.

A characteristic system of (40) is

$$
d p=\frac{d x^{0}}{k^{0}}=\frac{d x^{1}}{k^{1}}=\frac{d x^{2}}{k^{2}}=\frac{d x^{3}}{k^{3}}=-\frac{d S}{S A} .
$$

The integrals of the last system coincide partially with those for a characteristic conoid, and we suppose that the functions

$$
x^{\alpha}=x^{\alpha}\left(x_{0}^{\alpha}, k_{\alpha}^{0}, p\right), \quad k_{\alpha}=k_{\alpha}\left(x_{0}^{\alpha}, k_{\alpha}^{0}, p\right)
$$

have been found. By inserting their values into (42) and (43), we can write the integrals of (44) along bicharacteristics as

$$
\ln S=-\int\left(A_{0}+A_{1}\right) d p+\ln C\left(x_{0}^{\alpha}, k_{\alpha}^{0}\right),
$$

where $\ln C$ is an arbitrary constant along a bicharacteristic. Let us define

then we have

$$
\ln S_{0} \equiv-\int A_{0} d p
$$

$$
S\left(x_{0}^{\alpha}, k_{\alpha}^{0}, p\right)=C\left(x_{0}^{\alpha}, k_{\alpha}^{0}\right) S_{0}\left(x_{0}^{\alpha}, k_{\alpha}^{0}, p\right) \mathrm{e}^{-\int A_{1}(p) d p} .
$$

Here $S_{0}$ is a two-point scalar, $\Gamma$ is a null geodesic from $x_{0}$ to $x$. The boundary conditions consistent with (36), which also determine the integration constant $C$, are

$$
\text { i) } \begin{array}{r}
\lim _{p \rightarrow 0} S \tau=\frac{\sqrt{-\gamma\left(x_{0}\right)}}{\sqrt{g_{00}\left(x_{0}\right)-\gamma^{i k}\left(x_{0}\right) g_{0 i}\left(x_{0}\right) g_{0 k}\left(x_{0}\right)}}, \\
\gamma^{i k} g_{i !} \equiv \delta_{l}^{k}, \quad \gamma \equiv \operatorname{det} g_{i k}
\end{array}
$$

ii) $\lim _{p \rightarrow 0}\left(\tau \hat{M}^{*} S\right)$ is a limited function,

iii) $\lim \int\left(g^{i k} S\right)_{\mid k} d f_{i}=4 \pi$.

By eliminating $k_{\alpha}{ }^{0}$ and $p$ from (47) on the complete analogy of the case of the characteristic conoid, we get the Sobolev function $S\left(x, x_{0}\right)$.

3.5. Let us assume that $S$ is a known function. The second fundamental formula, the solution of integral equation (38) may be presented as follows:

$$
\begin{aligned}
& \varphi\left(x_{0}\right)=\sum_{n=0}^{\infty} \varphi_{n}\left(x_{0}\right) \\
& \varphi_{0}\left(x_{0}\right)=\int_{V_{1}}\left[S\left(x, x_{0}\right) \varrho(x)\right] d V+\frac{1}{4 \pi} \int_{f_{1}}\left[P^{i}(x)\right] d f_{i}, \\
& \varphi_{n}\left(x_{0}\right)=-\frac{1}{4 \pi} \int_{V_{1}}\left[\varphi_{n-1}(x) \hat{M}^{*} S\left(x, x_{0}\right)\right] d V
\end{aligned}
$$




\section{On the Physical Interpretation of the Fundamental Formulae}

4.1. In order to elucidate the physical content of the second fundamental formula, we shall consider a three-dimensional image of light propagation in an arbitrary reference frame by projecting a light cone onto the three-space $t=t_{1}=$ const. Fundamental formulae will also be valid on the future light cone $I^{+}\left(x_{0}\right)$, and instead of incoming radiation we shall consider the radiation issuing from point $x_{0}$. We shall still make use of Figure, assuming that the time direction has been inverted and the figure has been reversed.

Let us generalize the ordinary Huygens construction with the aim of analyzing the propagation of the field described by formula (52). Consider 1 adiation at the moment $t^{\prime}$ that lies between $t_{0}$ and $t_{1}$. The wave front is described through the projection $f^{\prime}$ of the intersection of three-space $t=t^{\prime}$ and the light cone $I^{+}\left(x_{0}\right)$ onto the three-space $t=t_{1}$. We shall call $f^{\prime}$, a quasisphere. Let us construct secondary waves, new quasispheres $f_{1}{ }^{\prime}$ with centres on the wave front of the primary waves. They are formed by the intersection of three-space $t=t_{1}$ with light cones $I^{+}\left(x^{\prime}\right)$. Taking an envelope of the quasispheres $f_{1}^{\prime}$ (in Figure only one quasisphere is indicated), we get a new wave front $f_{1}$. From formula (52) it follows that $\psi_{0}$ really has non-vanishing values at $t=t_{1}$ only on the quasisphere $f_{1}$. Now the Huygens principle can be paraphrased into a case of a curved space-time by replacing the term "spherical" with "quasispherical" in some well-known formulations, e. g., in that of $M$. Born and E. Wolf $\left[{ }^{11}\right]$. Thus we see that $\varphi_{0}$ satisfies the Huygens principle. Providing that $\hat{M}^{*} S=0, \varphi=\varphi_{0}$ and the whole solution satisfies the principle as well. The Huygens construction has been interpreted by A. Fresnel in terms of interference of secondary waves, its mathematical formulation has been given by G. Kirchhoff. The formula of the latter author is our first fundamental formula (38) with $S=\frac{1}{R}$ and $\hat{M}^{*} S=0$.

In case $\hat{M}^{*} S \neq 0$, the phenomenon of light propagation is more complicated, there is no complete interference of secondary waves inside quasispheres. Now the emission of secondary waves at point $x^{\prime}$ may also be considered as scattering of radiation at point $x^{\prime}$. From (53) it follows that one-fold scattering in $\varphi_{1}$ and $n$-fold scattering in $\varphi_{n}$ at all the points inside the quasisphere $f_{1}$ have been taken into account. It is interesting to note that not only the radiative field, but also the Coulomb field is scattered.

4.2. Further, we shall consider the influence of the curvature of spacetime on direct radiation $\varphi_{0}$. Note that $K$. Thorne and $S$. Kovács $\left[{ }^{12}\right]$ use the term "direct field" in the sense of the field that propagates from the source as if space-time were flat, while we include in this term the influence of the curvature on null geodesics.

Compared with the case of a flat space-time, there are three types of new phenomena to be taken into account in $\varphi_{0}$ :

i) direct influence of metric on sources;

ii) dependence of retarded time on metric (the "Shapiro effect");

iii) deflection of light-rays, which changes the inverse-square law for radiated energy.

The first phenomenon is taken into account immediately in sources, the second enters the fundamental formula via the relation $\left[\varphi\left(t, x^{i}\right)\right] \equiv$ $\varphi\left(t_{0}-\tau, x^{i}\right)$, the third is described by the Sobolev function itself. 


\section{The Maxwell Equations in the Schwarzschild Background}

To give an example of equation (1), let us consider the equations for an electromagnetic field in the Schwarzschild background. Instead of the equations for the four-potential $A^{\mu}$

$$
\begin{gathered}
\square A^{\mu}=4 \pi J^{\mu}, \\
J^{\mu} \equiv(\varrho, \vec{J})
\end{gathered}
$$

we generalize the following flat space-time equation:

$$
\square\left\{r\left(E_{r}+i H_{r}\right)\right\}=-2 \varrho-\vec{r} \vec{\nabla}_{\varrho}-\vec{r}_{, t}+-i \vec{r}(\vec{\nabla} \times \vec{J}),
$$

where $E_{r}$ and $H_{r}$ are the radial components of electric and magnetic field. Generalization is most naturally achieved by making use of the spinor formulation of the field equations given by $E$. Newman and R. Penrose ** $\left[{ }^{13}\right]$.

Let $l^{\mu}, t^{\mu}$ be real and $m^{\mu}, \bar{m}^{\mu}$ complex tetrad vectors $\left(\bar{m}^{\mu}\right.$ is a complex conjugate to $\mathrm{m}^{\mu}$ ) that satisfy the following orthogonality conditions:

$$
\begin{gathered}
l_{\mu} l^{\mu}=t_{\mu} t^{\mu}=m_{\mu} m^{\mu}=\bar{m}_{\mu} \bar{m}^{\mu}=0, \\
l_{\mu} t^{\mu}=-m_{\mu} \bar{m}^{\mu}=1, \\
l_{\mu} m^{\mu}=l_{\mu} \bar{m}^{\mu}=t_{\mu} m^{\mu}=t_{\mu} \bar{m}^{\mu}=0 .
\end{gathered}
$$

Let $F_{\mu \nu}$ be the tensor of the electromagnetic field. E. Newman and $\mathrm{R}$. Penrose define the components $\Phi_{A}$ of the spinor of the electromagnetic field:

$$
\begin{aligned}
& \Phi_{0}=F_{\mu v} l^{\mu} m^{v}, \\
& \Phi_{1}=\frac{1}{2} F_{\mu v}\left(l^{\mu} t^{v}+\bar{m}^{\mu} m^{v}\right), \\
& \Phi_{2}=F_{\mu v} \bar{m}^{\mu} t^{v} .
\end{aligned}
$$

In Bondi's coordinates $\left(u, r_{B}, \vartheta, \varphi\right)$ the Schwarzschild metric is

$$
d s^{2}=\left(1-\frac{2 m}{r_{B}}\right) d u^{2}+2 d u d r_{B}-r_{B}^{2}\left(d \theta^{2}+\sin ^{2} \vartheta d \varphi^{2}\right)
$$

and we can choose the tetrad vectors as follows:

$$
\left\{\begin{array}{l}
l_{\mu}=(1,0,0,0) \\
t_{\mu}=\left(\frac{1}{2}-\frac{m}{r_{B}}, 1,0,0\right) \\
m_{\mu}=-\frac{r_{B}}{\sqrt{2}}(0,0,1, i \sin \vartheta) .
\end{array}\right.
$$

The homogeneous wave equation for $\varphi \equiv r_{B} \Phi_{1}$ in the case of the line element $(54)$ has been given by several authors $\left[{ }^{14,15}\right]$. When we add the source terms, we have

** We note that the spinor formalism gives us separable equations for unknowns not only in the case of the Schwarzschild metric, but also in the case of the Kerr and Friedman backgrounds. 


$$
\begin{gathered}
\frac{2}{r_{B}}\left(r_{B} \dot{\varphi}\right)_{, r_{B}}-\Delta \varphi+\frac{2 m}{r_{B}^{2}}\left\{\left(r_{B} \varphi, r_{B}\right)_{, r_{B}}-\frac{1}{r_{B}} \varphi\right\}= \\
=\frac{1}{r_{B}}\left(r_{B}^{2} J \mu t_{\mu}\right)_{, r_{B}}+\frac{1}{\sqrt{2}} \partial_{-1}\left(J \mu \bar{m}_{\mu}\right),
\end{gathered}
$$

where the differential operator $\partial_{-1}$ is defined as follows:

$$
\partial_{-1} \equiv-\frac{1}{\sin \vartheta}\left(\frac{\partial}{\partial \vartheta}+\frac{i}{\sin \vartheta} \frac{\partial}{\partial \varphi}\right) \sin \vartheta
$$

When applying the Sobolev method, it is more convenient to use the isotropic coordinates $(t, r, \vartheta, \varphi)$ instead of Bondi's coordinates. Supposing that the Schwarzschild field is weak, and confining ourselves to the linear approximation ***, the corresponding coordinate transformation is

$$
\left\{\begin{array}{l}
u=t-r\left(1+\frac{m}{r}\right)-2 m \ln (r-m), \\
r_{B}=r\left(1+\frac{m}{r}\right)
\end{array}\right.
$$

and it changes equation (55) to the following form:

$$
\begin{aligned}
& \left(1+\frac{2 m}{r}\right) \varphi, t t-\left(1-\frac{2 m}{r}\right) \Delta \varphi-\frac{2 m}{r^{3}} \varphi= \\
= & \left(1-\frac{m}{r}\right)\left\{J^{0}+\frac{r}{2} J^{0}{ }_{, r}+\frac{r}{2}\left(1+\frac{4 m}{r}\right) J^{r}, t\right\}- \\
- & \frac{i r}{2 \sin \vartheta}\left(1+\frac{m}{r}\right)\left\{\left(\sin ^{2} \vartheta J^{\varphi}\right)_{, 0}-J^{\vartheta}{ }_{, \varphi}\right\}+0\left(m^{2}\right) .
\end{aligned}
$$

The Sobolev function for the last equation is very simple:

$$
\begin{gathered}
S\left(x, x_{0}\right)=\left(1+\frac{2 m}{r}\right) \cdot \frac{1}{R}+0\left(m^{2}\right), \\
R^{2} \equiv\left(x^{1}-x_{0}^{1}\right)^{2}+\left(x^{2}-x_{0}^{2}\right)^{2}+\left(x^{3}-x_{0}^{3}\right)^{2}, \\
\left\{\begin{array}{l}
x^{1} \equiv r \sin \vartheta \cos \varphi, \\
x^{2} \equiv r \sin \vartheta \sin \varphi, \\
x^{3} \equiv r \cos \varphi .
\end{array}\right.
\end{gathered}
$$

After the evaluation of $\varphi$ the remaining spinor components can be calculated from the Maxwell equations.

If we consider asymptotic radiation far from the Schwarzschild mass, only $\Phi_{2}$ is of importance. It can be calculated from the following equation (see $\left.\left[{ }^{16}\right]\right)$ :

$\delta_{-1} \Phi_{2}=-\frac{r}{\sqrt{2}}\left\{\Phi_{1, t}-\frac{1}{r^{2}}\left(r^{2} \Phi_{1}\right)_{, r}+\frac{m}{r}\left(\Phi_{1, t}+\frac{1}{r^{2}}\left(r^{2} \Phi_{1}\right)_{, r}+\frac{2}{r} \Phi_{1}\right)\right\}$.

The integration of the above equation is rather simple if we introduce the stereographic coordinates $(\zeta, \bar{\zeta})$ instead of the polar angles $(\vartheta, \varphi)$ :

$$
\zeta=\cot \frac{\vartheta}{2} \mathrm{e}^{i \varphi}
$$

*** Formulae for the exact theory are given in $\left[{ }^{16}\right]$. 
In this case we have

$$
\partial_{-1} \equiv \frac{(1+\zeta \bar{\zeta})^{2}}{\bar{\zeta}} \frac{\partial}{\partial \zeta} \frac{\sqrt{\zeta \bar{\zeta}}}{1+\zeta \bar{\zeta}}
$$

and the evaluation of $\Phi_{2}$ reduces to one integration over $\zeta$.

\section{REFERENCES}

1. $\mathrm{H}$ a d a mard J., Lectures on Cauchy's problem in linear partial differential equations, Yale University Press, New Haven, 1923.

2. De Witt B. S., B rehme R., Ann. Phys., 9, 220 (1960).

3. S o b o le f f S., Матем. сб. (Recueil Mathématique), 1 (43), No. 1, 39 (1936).

4. Chevali er M.. Ann. Inst. H. P o inc a ré, A12, 71 (1970).

5. Соболев С. Л., Уравнения математической физики, М., 1966.

6. Morette D e W it t C., D e W it t B. S., Physica, 1, 3 (1964).

7. Peters P. C., Phys. Rev., 146, 938 (1966); D7, 368 (1973).

8. Lichnerowicz A., Théories relativistes de la gravitation et de l'électromagnétisme, Paris, 1955.

9. Фок В. А., Теория пространства, времени и тяготения, М., 1961.

10. C a ric a to G., Rend. di Math., 22, 416 (1963).

11. Борн М., В ольф Э., Основы оптики. М., 1970.

12. Thorne K. S., Kovács S. J., OAP-384 (Preprint) (1974).

13. N ewm an E. T., Pen rose R., J. Math. Phys., 3, 566 (1962); 4, 998 (1963).

14. B a rdeen J., Press W., J. Math. Phys., 14, 7 (1973).

15. Ун т В., Керес П., Изв. АН ЭССР, Физ. Матем., 21, 17 (1972).

16. К у уск П., Канд. дис., Тарту, 1974.

Academy of Sciences of the Estonian SSR,

Institute of Astrophysics and Atmospheric Physics,

Received Institute of Physics

Dec. 26,1975

V. UNT, Piret KUUSK

\section{UUS MEETOD LAINETE TEKKE JA LEVIKU ARVUTAMISEKS KÕVERAS AEGRUUMIS}

Esitatakse uus formalism lainete arvutamiseks kõveras aegruumis. On tuletatud integraalvõrrand. mis on ekvivalentne muutuvate kordajatega lainevõrrandiga, ja analüüsitud tema füüsikalist sisu. Uus formalism on rakendatav meelevaldsetes koordinaadisüsteemides.

\section{В. УНТ, Пирет КууСК}

\section{НОВЫИ МЕТОД ДЛЯ РАСЧЕТА ГЕНЕРАЦИИ И РАСПРОСТРАНЕНИЯ ВОЛН В ИСКРИВЛЕННОМ ПРОСТРАНСТВЕ-ВРЕМЕНИ}

Путем модифицирования метода Соболева для решения проблемы Коши выработан новый формализм для расчета генерации и распространения волн в искривленном пространстве-времени. Дано интегральное уравнение, эквивалентное волновому уравнению с переменными коэффициентами, и обсуждено его физическое значение. Новый формализм применим во всех допустнмых системах координат. 